\title{
Recurrent Colorectal Adenocarcinoma
}

National Cancer Institute

\section{Source}

National Cancer Institute. Recurrent Colorectal Adenocarcinoma. NCI Thesaurus. Code C162441.

The reemergence of colorectal adenocarcinoma after a period of remission. 